\title{
Estudio preliminar del cerro del castillo de Montefrío (Granada)
}

\author{
Preliminary study of the Hill of the Castle of Montefrío (Granada)
}

\author{
Rafael Moreno Guerrero a, Luis José García-Pulido ${ }^{\text {b }}$ \\ a Departamento de Arte y Arquitectura - Universidad de Málaga, Málaga, Spain, rafaelmoreno@uma.es

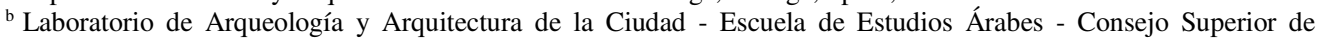 \\ Investigaciones Científicas, Granada / Departamento de Arte y Arquitectura - Universidad de Málaga, Málaga, Spain, \\ luis.garcia@uma.es
}

\begin{abstract}
The castle of Montefrío (Granada) was one of the fortresses that formed the last line of defense of the Nasrid kingdom. After its surrender, in 1486, the castle served as a Castilian border stronghold until the fall of the Nasrid capital, Granada, six years later, which put an end to the Christian conquest of al-Andalus.This work tries to analyze the evolution of the hill were the castle is, from the implantation of the Nasrid fortress to the present day, through the continuous transformations from a citadel, a military fortress, a church and, today, a centre of interpretation. This place is a territorial and landscape landmark that has shaped the environment of Montefrío and has been a key piece in its history and in its urban development. The study focuses on the analysis of the evolution of the constructive techniques developed by the Nasrid and the Castilian for the defense of this stronghold, through the archaeological remains preserved in the site. The preliminary study of this castle is a starting point for a deeper investigation that will be extended to other fortresses in the mountainous region between Córdoba and Granada. The study of the castle of Montefrío is therefore a methodological approach that will serve as the basis for a more extensive territorial research.
\end{abstract}

Keywords: Nasrid Kingdom, fortification, construction techniques, medieval border, poniente granadino.

\section{Introducción}

En este trabajo se recogen, a modo de resumen, los datos de partida para el estudio y caracterización de las técnicas constructivas utilizadas en el castillo de Montefrío y las estructuras emergentes que lo rodean. El objetivo es la caracterización y puesta en valor de los elementos que hoy se conservan en el cerro del castillo, pertenecientes a una fortaleza clave para la defensa del reino nazarí de Granada durante los siglos XIII al XV. Se estudia este castillo desde un punto de vista histórico, arquitectónico y territorial, a partir de las estructuras que hoy se mantienen en pie -muy modificadas por las transformaciones que se llevaron a cabo tras la conquista cristiana y el abandono del recinto fortificado en Época Moderna- y los estudios previos publicados, principalmente centrados en la época nazarí.

La investigación abarca las transformaciones que han sufrido sus estructuras desde su concepción como castillo andalusí, pasando por la fortificación del castillo en el momento en el que Montefrío se convierte en una población de frontera con el reino de Castilla y por las transformaciones que se dan tras la conquista cristiana, primero para reconfigurarla como una fortaleza abaluartada, posteriormente para implantar en ella una iglesia 


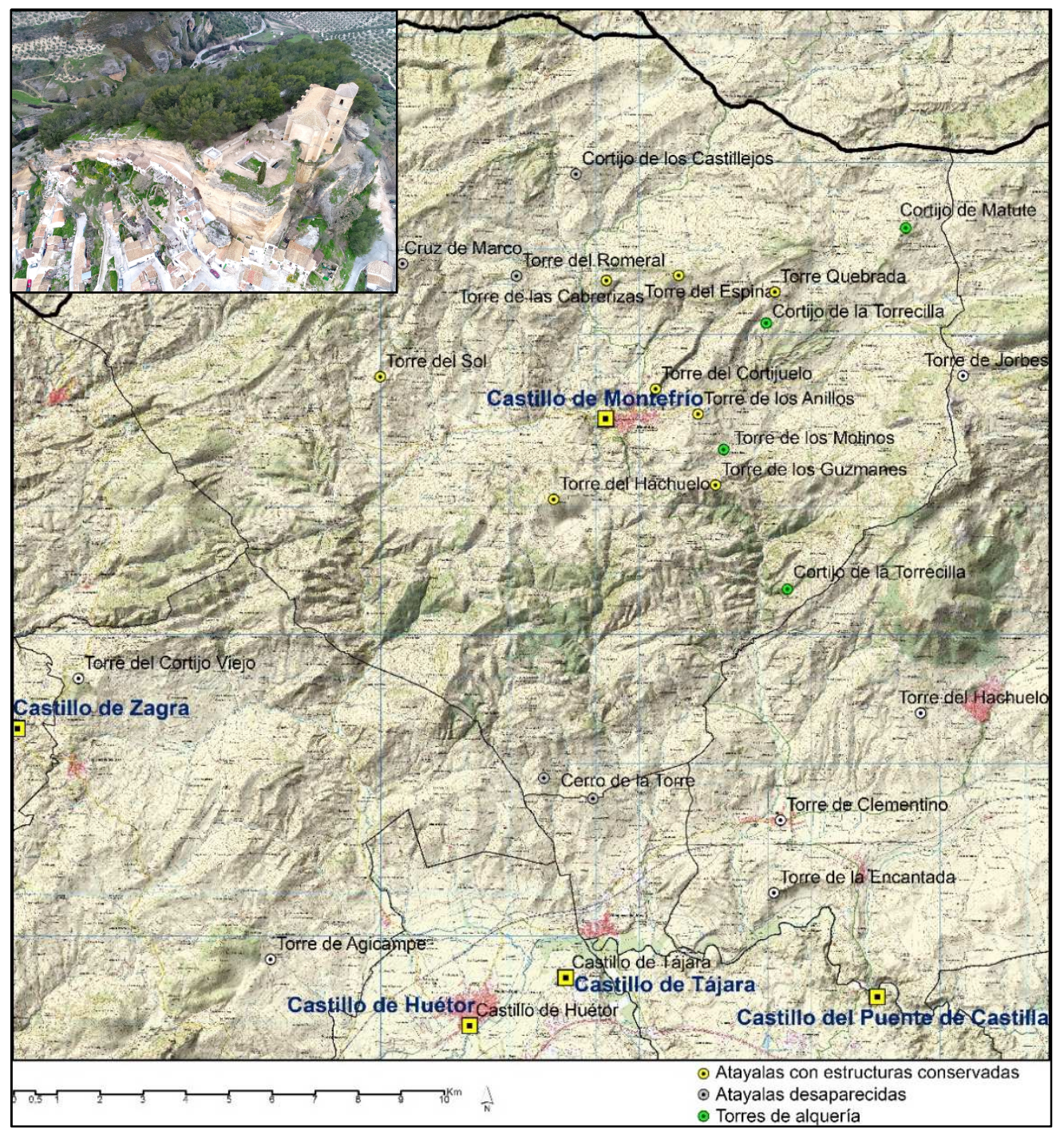

Fig. 1. Ubicación de las distintas estructuras defensivas del territorio de Montefrío.

renacentista y, finalmente, con su abandono en favor de los nuevos crecimientos urbanos del municipio.

\section{El sistema de atalayas en torno a Montefrío}

Los sistemas defensivos de Montefrío en la Edad Media se configurarían en la etapa que iría de 1264 a 1369 , caracterizada por el avance y retroceso de la línea de frontera con los reinos de Sevilla y Jaén (Pedregosa Megías, 2014, Torre Espinosa, pp. 155-175). Tras la Batalla del Salado o del Estrecho (1340), Alfonso XI (1311-1350) conquistó Alcalá la Real, Castillo de Locubín, Priego, Rute y Benamejí en 1341 (Jiménez Puertas, 2002, p. 147), lo que hizo retroceder la frontera noroccidental del Reino de Granada, quedando Montefrío como uno de los castillos nazaríes más adelantados.

Ante esta situación, Muḥammad V (13541359/1362-1391) fomentaría la refortificación y construcción de nuevas estructuras defensivas, reorganizando la frontera, tal y como recogería Ibn 
al-Jațīb en la Ihāața (Bordes García, 2001, p. 71). Para ello, se trataron de controlar los pasos entre las zonas que ya estaban en poder de la corona de Castilla en la vertiente norte de la Cordillera Penibética y la capital nazarí. También se habría favorecido la edificación de torres para proteger de las cabalgadas cristianas a las comunidades campesinas de algunas alquerías. Se creaba, de esta manera, una línea fronteriza en el sector noroccidental del reino de Granada, con las fortalezas de Montefrío, Íllora y Moclín, apoyadas por una serie de torres y atalayas.

En el término municipal de Montefrío podrían haber existido unas 18 torres de distinta naturaleza (Fig. 1). De ellas, al menos 3 podrían haber pertenecido a alquerías, entre las que estarían la "Torre Nunes", que, aunque el topónimo no se ha conservado, aparece citada en la Crónica del Condestable Miguel Lucas de Iranzo ${ }^{1}$ (Soriano, 1993, pp. 198-199). Otras torres más de esta tipología podrían haber existido en los cortijos de Matute, el Cortijo de la Torrecilla en la zona conocida como La Torrecilla, y la de otro cortijo del mismo nombre que aparece muy cerca de Torre Quebrada y El Castellón. Las atalayas que habrían definido un anillo en torno a Montefrío si situarían a un radio máximo de $6 \mathrm{~km}$, estando constituidas por al menos 13 torres denominadas: Cruz de Marco (desparecida), del Romeral o del Rosal (desaparecida), del Sol, Hachuelo, Guzmanes, Cabrerizas, Espinar, Quebrada, Anillos, Cortijuelo, Castillejos. Habría una o dos más en los promontorios denominados Cerro de la Torre, situados al sur del término de Montefrío, cerca del límite municipal con Villanueva de Mesía. También aparece el topónimo Torre de los Molinos en las inmediaciones de la Torre de los Guzmanes, que podría haber desempeñado este cometido, al controlar el valle donde éstos se situaban. Los datos que permiten identificarlas proceden de las prospecciones e intervenciones arqueológicas, las fuentes históricas, la toponimia conservada y la información oral. Estarían situadas en zonas altas o abruptas, en cerros elevados que permitían la comunicación directa frente a las correrías cristianas. Tenían, por tanto, una clara función de vigilancia y control del territorio, avisando a la población rural indefensa de las venidas cristianas desde el otro lado de la frontera (Pedregosa Megías, Rivera Groennou, 2013, p. 158).

Actualmente se conservan restos materiales visibles de 7 torres: las del Sol, Hachuelo, Cabrerizas, Espinar, Anillos, Guzmanes y Cortijuelo, de las que se tiene certeza de su ubicación geográfica. Por su parte, la Torre Quebrada se encuentra enmascarada por grandes majanos que no permiten identificar ninguna de sus caras. Del resto no quedan elementos constructivos evidentes, pudiendo existir restos de su cimentación, hecho que habría que comprobar arqueológicamente (Pedregosa Megías, 2011, p. 64).

Estas atalayas presentan patrones comunes en cuanto a su posicionamiento, técnicas constructivas, y materiales utilizados, independientemente de la zona en la que se construyesen. Están edificadas con mampuestos dispuestos regularmente y distribuidos en hiladas horizontales, que quedan enmarcados entre verdugadas de ripios y lajas. Todo ello parece apuntar al seguimiento de un programa constructivo, al tratarse de una obra promovida por el poder (Acién Almansa, 1999, p. 437; Luque, 2003, pp. 37 y 82; Malpica Cuello, 1998, pp. 273; Malpica Cuello, 2003, p. 139).

\section{La frontera norte de Granada con el reino de Jaén}

Los estudios arqueológicos realizados sobre las técnicas constructivas utilizadas en los castillos y torres-atalayas de la frontera norte del reino de Granada (Montefrío, Íllora, Colomera, Iznalloz, Píñar, Montejícar y Moclín) han venido a clasificarlas en tres (Alawna, 2004), que corresponderían a otras tantas fases diferentes: dos de ellas asociadas a época andalusí, que son el tapial y la mampostería concertada con verdugadas de lajas; y una última de época castellana, la técnica de sillería, que está presente en la parte superior de la fortaleza de Montefrío, mostrando las transformaciones que sufrió la alcazaba tras la conquista.

El estudio de la evolución de esta frontera norte con el reino de Jaén, caracterizada por zonas montañosa que ya contaban con numerosas fortificaciones antes de convertirse en banda fronteriza, aporta algunas claves importantes para entender el contexto de una frontera militar en con- 
tinuo movimiento, cuya evolución queda reflejada en las técnicas constructivas de cada fortaleza, ampliamente abordada por la bibliografía desde la segunda mitad del siglo XX, desde las que recogen una breve descripción de cada castillo y fortaleza por orden alfabético y con representaciones gráficas sencillas de los más relevantes (Morales Talero, 1958; Perea Carpio, 1983; Vidal Castro, 200; Eslava Galán, 2015), a las más recientes que han hecho visibles, de forma digital, algunos de los bienes patrimoniales más significativos de la provincia de Jaén desde el siglo IV hasta el siglo XV (Castillo Armenteros, et al., 2019),

\section{La frontera con el reino de Sevilla}

El reino hispalense tuvo que reforzar sus fronteras debido a que, tras su incorporación a Castilla, la rápida conquista del valle del Guadalquivir a principios del siglo XIII ocasionó la creación de dos nuevas líneas fronterizas: la de Portugal por el oeste, y la de Granada por el sureste (Molina Rozalem, 2017). Las técnicas constructivas de defensa que se empiezan a desarrollar en esta época son las mismas que se realizarán al lado contrario de la frontera, en el reino nazarí, y algunas de las fortalezas que se crean en el siglo XIII irán ganando después protagonismo en la ofensiva final contra Granada.

Así, las modificaciones introducidas en el castillo de Montefrío después de 1486, seguirán los patrones y las técnicas constructivas desarrolladas en las fortificaciones castellanas de ese momento.

\section{El cerro del castillo de Montejícar}

Más allá de los valores patrimoniales materiales, el cerro del castillo de Montefrío constituye hoy un elemento configurador del territorio y un hito paisajístico de primer nivel.

Desde que en 2015 fuera reconocido por la revista National Geographic como uno de los 10 pueblos con las mejores vistas del mundo ${ }^{2}$, la demanda cultural de Montefrío han aumentado de forma considerable, lo que incentivó la mejora del material de promoción de sus bienes patrimoniales. En 2010 ya se había realizado una recreación virtual en 3D del castillo de Montefrío (Valdivia
García, 2010; Pedregosa Megías, 2011) para ser utilizada como material de difusión en la Iglesia de la Villa, el templo cristiano que se levantó sobre los restos del castillo nazarí y que hoy se ha adaptado como centro de interpretación (Fig. 2).

El castillo de Montefrío guarda elementos comunes con el resto de fortalezas de su entorno, bien por ser coetáneas o bien por haber tenido un uso similar y estar en conexión con ésta (Ruiz Fernández de Cañete, 2008). En él se conservan estructuras de época nazarí (Fig. 3), como ocurre en fortalezas cercanas como las de Algarinejo, Alhama de Granada o Huétor Tájar.

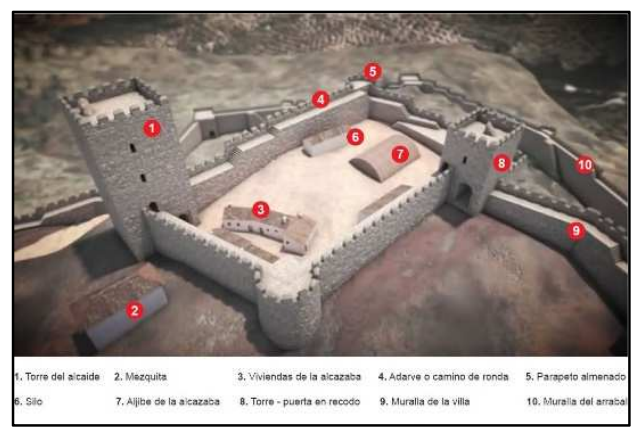

Fig. 2. Recreación de la alcazaba nazarí de Montefrío (Valdivia, 2010).

La relevancia de Montefrío como enclave paisajístico y su importante papel durante época nazarí hacen que buena parte de la bibliografía publicada sobre la localidad tenga que ver, principalmente, con su papel histórico en la Edad Media y con realidades o valores vinculados al paisaje y al interés turístico que éste ha generado.

Los trabajos que tienen como objeto principal el cerro del castillo, la destacan como elemento estratégico en la organización territorial del reino de Granada y, en especial, de su relevancia en el sistema defensivo de torres vigía, castillos y atalayas existentes a nivel territorial. Así, el Centro de Estudios Paisaje y Territorio $^{3}$ destaca los montes occidentales granadinos como "uno de los escenarios con mayor concentración de torres, atalayas, fortalezas y castillos por kilómetro cuadrado del mundo" (VV.AA., 2015). 


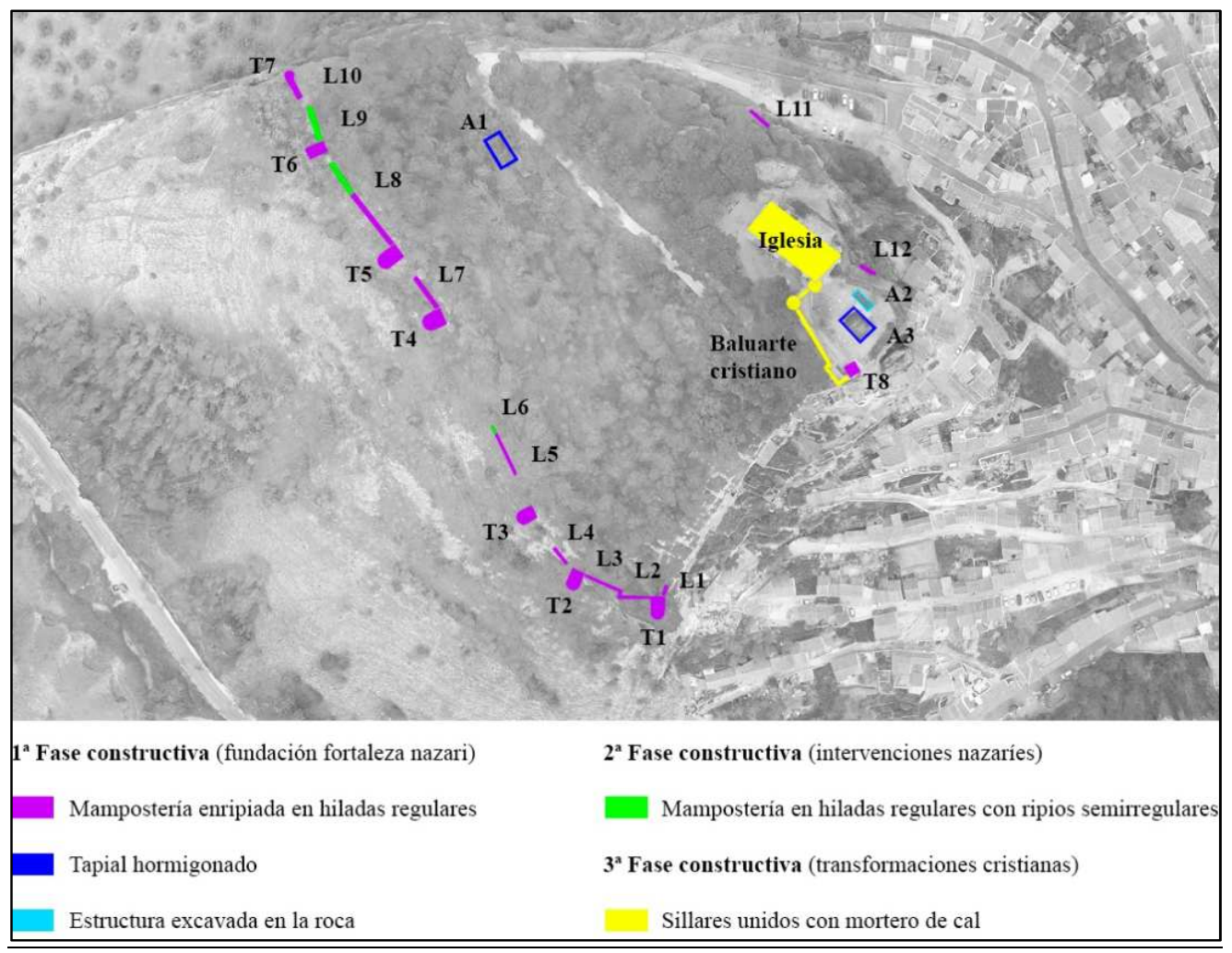

Fig. 3. Técnicas constructivas identificadas en el castillo de Montefrío (a partir de Mattei, 2013).

Con respecto a las publicaciones científicas que se centran en exclusiva en el estudio histórico de Montefrío, uno de los autores que más trabajos ha dedicado a la localidad es el historiador y arqueólogo Rafael Jesús Pedregosa Megías, cuyas aportaciones van desde una visión general de la historia de Montefrío hasta un acercamiento a elementos concretos. Dicho autor ha realizado una investigación sobre la villa en época nazarí (Pedregosa Megías, 2011) y su posterior evolución tras la conquista castellana (Pedregosa Megías, 2012), así como varios trabajos sobre intervenciones arqueológicas en las torres-atalayas (Bellón Aguilera, Pedregosa Megías, 2007) y sus técnicas constructivas (Pedregosa Megías, Rivera Groennou, 2013), además de un estudio sobre los restos cerámicos medievales que se hallaron en el castillo de Montefrío (Pedregosa Megías, 1990). Especialmente relevantes son sus aportaciones en cuanto al estudio, caracterización y propuestas de mejora de las torres-vigía, dado que son éstas las que hacen de la frontera granadina un sistema territorial de defensa, articulándolo y dando sentido a la función de los castillos como fortificaciones principales.

\section{Técnicas constructivas en el castillo de Mon- tefrío}

En el castillo de Montefrío se reconocen cinco técnicas constructivas bien diferenciadas, a partir de las estructuras conservadas:

1. Mampostería enripiada en hiladas regulares. Aparece en las 8 torres que se conservan de época nazarí, así como en la mayoría de tramos de muralla exterior que las une (Fig. 4). Por norma general, los mampuestos están dispuestos en hiladas separadas, tomadas con mortero de cal y separadas con verdugadas de piedra.

2. Mampostería en hiladas regulares con ripios semirregulares. Esta técnica sólo aparece en tres tramos de muralla exterior, lo que parece indicar que 
estos tramos fueron reparaciones o reconstrucciones hechas con posterioridad. Los mampuestos presentan distintas dimensiones y disposición, conformando lienzos muy heterogéneos que los diferencian del resto de muralla.

3. Tapial hormigonado. Es la técnica utilizada en dos grandes aljibes, uno de ellos dentro de la alcazaba y el otro en el recinto de la villa. Ambos conservan parte de sus muros de tapial hormigonado, además del arranque de las bóvedas que sirvieron para su cubierta.

4. Estructuras excavadas en la roca. El otro de los aljibes que se encuentra en la alcazaba, de menores dimensiones, está totalmente excavado en la roca y no conserva restos de su cubrición.

5. Sillares unidos con mortero de cal. Esta es la técnica constructiva que utilizaron los cristianos tras la toma del castillo, utilizándola para construir el fortín sobre la alcazaba original. Se trata de una muralla de sillería tallada unida con mortero de cal, en la que destacan las troneras de artillería. Además, posteriormente también se construiría la Iglesia de la Villa con sillares de gran tamaño.

\section{Conclusiones}

Hasta ahora se han publicado trabajos sobre las fortificaciones castellanas y nazaríes existentes a uno y otro lado de la frontera definida por la subbética cordobesa y el poniente granadino entre los siglos XIII y XV. Sin embargo, no se ha abordado este proceso considerando esta banda fronteriza como un elemento permeable, sino estanco, por lo que se hace necesario llevar a cabo este estudio desde una visión más amplia e integradora sobre las construcciones desarrolladas en esta franja por ambas sociedades.

Las fortificaciones conservadas en la comarca de los Montes Occidentales de Granada y las localidades limítrofes de la subbética cordobesa cuentan con diversas actuaciones y estudios particulares. Sin embargo, es necesario realizar un trabajo de unificación de esta información, complementada con otra nueva que pueda obtenerse a partir de la documentación material de las estructuras conservadas. Ello permitirá llevar a cabo estudios comparativos y el análisis territorial de la implantación de estas alcazabas. Así, por ejemplo, una característica común son las transformaciones que sufrieron estas fortificaciones durante el siglo $\mathrm{XV}$, cuando fueron conquistadas por las tropas castellanas y tuvieron que ser rápidamente adaptadas a las nuevas técnicas y tácticas poliorcéticas.

Partiendo de los datos que tenemos hasta el momento, debe realizarse un doble análisis constructivo y territorial que permita tanto estudiar y caracterizar los sistemas constructivos empleados en la arquitectura defensiva del reino nazarí, como el estudio comparado de las estructuras emergentes en el entorno de las fortificaciones objeto de estudio. Un estudio así podrá definir las aportaciones e influencias que se realizaron desde ambos lados de la frontera y permitirá establecer las dinámicas territoriales y los valores paisajísticos de esta zona montañosa, realizando el diagnóstico del estado actual de conservación de cada elemento constructivo estudiado. Este estudio debe llevarse a cabo desde una perspectiva y un análisis crítico que tenga en cuenta los criterios utilizados en las intervenciones realizadas, para

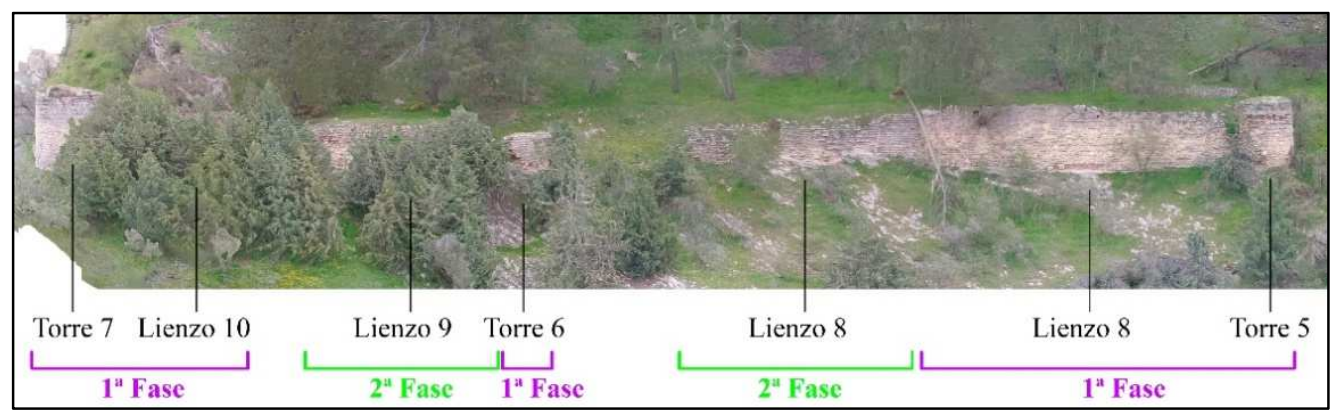

Fig. 4. Alzado del tramo T5-T7, con lienzos de muralla de la $1^{\mathrm{a}}$ y $2^{\mathrm{a}}$ fase constructiva. 
poder definir las estrategias más idóneas para su mantenimiento, conservación y/o rehabilitación.

\section{Notas}

${ }^{1}$ Entre los años 1455-1463 se generalizaron las campañas castellanas en la zona de Montefrío, durante el reinado del monarca castellano Enrique IV (1425-1474), y las cabalgadas del Condestable en 1463-1464.

${ }^{2}$ A ello han contribuido dos autores japoneses: el fotógrafo y profesor de la Universidad de Yokohama, Yuri Oyma, y el escritor Osamu Takeda, (Takeda, 1999).

${ }^{3}$ En él se incluye la Consejería de Medio Ambiente y Ordenación del Territorio de la Junta de Andalucía y las Universidades Públicas andaluzas.

\section{Agradecimientos}

Este trabajo ha sido realizado como parte de la investigación doctoral de Rafael Moreno Guerrero $y$ en el marco del Proyecto I+D+i "Las atalayas que defendieron el reino de nazarí de Granada. Análisis y documentación científica (Nazalaya)" (Programa Estatal de Fomento de la Investigación Científica y Técnica de Excelencia del Ministerio de Economía, Industria y Competitividad, HAR2016-79689-P, 2017-2020), convocatoria de 2016, cofinanciado con fondos FEDER; así como en el proyecto I+D+i en el marco del Programa Operativo Feder Andalucía 2014-2020, de la Convocatoria de la Universidad de Málaga para proyectos retos y frontera, convocatoria de 2018, titulado "Documentación gráfica de los castillos y alcazabas medievales conservados en Andalucía. Puesta al día del conocimiento y difusión de este legado patrimonial" (UMA18-FEDERJA257).

\section{Bibliography}

Acién Almansa, M. (1999). "Los tugur del reino nazarí. Ensayo de identificación”, Castrum, 5, Archéologie des espaces agraries mediterranées au Moyen Age, Murcia, pp. 427-438.

Alawna, S. (2004). Las técnicas constructivas empleadas en los castillos de la frontera norte del reino de Granada, Tesis doctoral de Universidad de Granada, Granada.

Bellón Aguilera, J.; Pedregosa Megías, R.J. (2007). “En la frontera del reino de Granada: una intervención arqueológica en las torres-atalayas de Montefrío", Antiquitas, 16, pp. 121-130.

Bordes García, S. (2001). "La frontera nazarí en el sector oriental de Loja: Íllora y Montefrío”, in López López, M., ed., La última frontera de al-Andalus. (Guía arqueológica del poniente granadino), Loja, pp. 55-75.

Castillo Armenteros, J.C., et al. (2019). "El patrimonio medieval de Jaén en Europeana. El proyecto Europeana Archaeology", Arqueología y territorio medieval, 26, pp. 275-284.

Eslava Galán, J. (2015). Príncipes, castillos y batallas en el paraíso disputado (un paseo por la historia y la arqueología de Jaén), Instituto de Estudios Giennenses, Jaén.

Jiménez Puertas, M. (2002). El poblamiento de Loja en la Edad Media, Chrónica Nova, Estudios Históricos, 77, Universidad de Granada, Granada.

Jiménez Puertas, M.; Mattei L. (2014). El patrimonio arqueológico medieval del poniente granadino: (Comarcas de Alhama, Loja y los Montes Occidentales), Ed. Alhulia, Granada.

Luque Martínez, F. De. (2003). El Castillo de Píñar: Análisis estratigráfico de las estructuras de superficie, Ed. Nakla, Granada.

Malpica Cuello, A. (1998). "Los Castillos en época nazarí. Una primera aproximación”, in Castillos y territorio en alAndalus, Granada, pp. 246-293.

Malpica Cuello, A. (2003). Los Castillos en Al-Andalus y la organización del territorio, Universidad de Extremadura, Cáceres.

Martín García, M.; Bleda Portero, J.; Martín Civantos, J.M. (1999). Inventario de arquitectura militar de la provincia de Granada (siglos VIII al XVIII), Diputación de Granada, Granada. 
Mattei, L. (2013). Los castillos de frontera nazaríes y sus precedentes en los montes occidentales de Granada. Un análisis espacial y del territorio, Tesis doctoral de la Universidad de Granada, Granada.

Molina Rozalem, J.F. (2017). Fortificaciones de la banda morisca en la provincia de Sevilla, Diputación Provincial de Sevilla, Sevilla.

Morales Talero, S. de (1958). “Castillos y murallas del Santo Reino de Jaén”, Boletín del Instituto de Estudios Giennenses, 18, pp. 9-83.

Pedregosa Megías, R.J. (1990). "El castillo de Montefrío (Granada): la cerámica medieval de superficie”, Antiquitas, 17, pp. 149-156.

Pedregosa Megías, R.J. (2011). Guía histórico-arqueológica del castillo y atalayas de Montefrío (Granada), Sevilla.

Pedregosa Megías, R.J. (2012). "La evolución de una villa nazarí de frontera: Montefrío. Antecedentes, configuración y transformación tras la conquista castellana”, Revista del Centro de Estudios Históricos de Granada y su Reino, 24, pp. 73-103.

Pedregosa Megías, R.J.; Rivera Groennou, J.M. (2013). “Técnicas constructivas en las torres atalayas de Montefrío (Granada): La Torre de Las Cabrerizas", Arqueología y Territorio Medieval, 20, pp. 155-175.

Peinado Santaella, R.G. (1981). "Financiación de la guerra y señorialización del Reino de Granada: Montefrío y la Casa de Aguilar", Baética: Estudios de arte, geografía e historia, 4, pp. 167-192.

Perea Carpio, C. (1983). "La frontera concejo de Jaén - Reino de Granada en 1476”, Cuadernos de Estudios Medievales y Ciencias y Técnicas Historiográficas de la Universidad de Granada, 10-11, pp. 231-238.

Ruiz Fernández de Cañete, J. (2008). La frontera nazarí. Castillo-Fortaleza de Montefrío, Caja Granada, Granada.

Soriano, C. (1993). Los hechos del Condestable don Miguel Lucas de Iranzo, Estudio y edición de C. Soriano, Universidad Complutense, Madrid.

Takeda, O. (2011). Los 100 pueblos más bellos de España, Seiquer, Madrid.

Torres Balbás, L. (1949). “Arte almohade. Arte nazarí. Arte mudéjar”, Ars Hispaniae, Historia Universal del Arte Hispánico, Madrid, vol. 4.

Valdivia García, M. (2010). "Una mirada virtual al pasado: recreación del castillo de Montefrío en época nazarí”, Virtual Archaeology Review, vol. 4, no. 8, pp. 9-14.

Vidal Castro, F. (2001). "Sierra Mágina dividida: formación y desaparición de la frontera nazarî', Sumuntán: anuario de estudios sobre Sierra Mágina, 15, pp. 11-38.

VV.AA. (2015). Montes occidentales. Catálogo de paisajes de la provincia de Granada, Centro de Estudios Paisaje y Territorio, Consejería de Medio Ambiente y Ordenación del Territorio, Sevilla. 